\title{
Differential glycosylation of envelope gp120 affects reactivity with HIV-1 specific antibodies
}

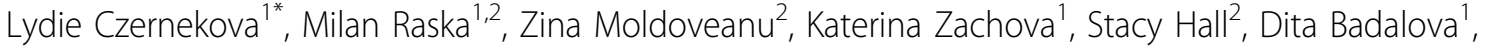 \\ Alzbeta Krcmarska', Hana Synkova', Michael Hoelscher ${ }^{3}$, Leonard Maboko ${ }^{4}$, Rhubell Brown², Zdenek Novak², \\ Jiri Mestecky², Jan Novak²
}

From Abstracts from International Symposium HIV and Emerging Infectious Diseases 2014 Marseille, France. 21-23 May 2013

\section{Introduction}

Cellular entry of human immunodeficiency virus type 1 (HIV-1) depends on envelope glycoprotein (gp120/gp41) interactions with host-cell receptors. Approximately onehalf of molecular mass of gp120 consists of N-glycans which may act as antigenic determinants as well as a shield against immune recognition. We have shown that glycosylation of subtype B HIV-1 gp120 varies according to the producing cell type and the differential glycosylation affects reactivities with serum antibodies of persons infected with HIV-1 subtype B. Here we studied reactivities of above proteins with panel of monoclonal gp120specific broadly neutralizing antibodies and sera from persons infected with HIV-1 subtype A/C.

\section{Materials and methods}

Recombinant gp120 produced in different cell lines (HEK 293T, Jurkat, RD, HepG2, and $\mathrm{CHO}$ ) were tested as a native and after partial removal of $\mathrm{N}$-glycans by PNGase $\mathrm{F}$ under native conditions. Several methods (ELISA, Cytometric Bead Array - CBA, SDS-PAGE with western blot, and dot blot) were used for determination and comparison of reactivities with monoclonal gp120-specific antibodies (268-D IV, F425 B4e8, 257-D IV, 447-52D, 19b, 2G12, and b12) or with sera of HIV-1 subtype A/C-infected persons.

\section{Results}

After partial removal of N-glycans from gp120, the reactivity of most monoclonal antibodies increased, as did the reactivities of sera from HIV-1-infected persons. The largest increase in binding of polyclonal antibodies after PNGase F treatment was found for gp120 expressed in
HEK $293 \mathrm{~T}$ and CHO cell lines, common producers of recombinant proteins for vaccination purposes. Conversely, the gp120 produced by $\mathrm{T}$ cells (Jurkat) displayed the least increase in reactivity after partial deglycosylation.

\section{Conclusion}

Changes in reactivity of selected monoclonal antibodies with native and PNGase F-treated proteins indicated that some glycans were resistant to deglycosylation and that this characteristic was dependent on producer cell type. Furthermore, CBA allowed more sensitive detection (about 40-times) of gp120-specific antibodies compared to ELISA.

Supported by CZ.1.07/2.3.00/20.0164 European Social Fund, UAB CFAR Developmental Grant (P30AI027767) and a Pilot Grant from UAB School of Medicine.

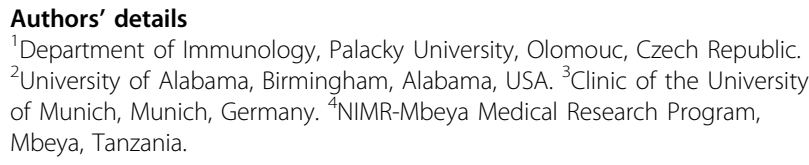

doi:10.1186/1471-2334-14-S2-P68

Cite this article as: Czernekova et al.: Differential glycosylation of envelope gp120 affects reactivity with HIV-1 specific antibodies. BMC Infectious Diseases 2014 14(Suppl 2):P68. 